\title{
Volume of Geodesic Balls in Finsler Manifolds of Hyperbolic Type
}

\section{Carlos Ogouyandjou}

Institut de Mathematiques et de Sciences Physiques (IMSP), Universite d'Abomey-Calavi, Porto-Novo, Republic of Benin

Email: ogouyandjou@imsp-uac.org

Received 20 June 2014; revised 20 July 2014; accepted 28 July 2014

Copyright (C) 2014 by author and Scientific Research Publishing Inc.

This work is licensed under the Creative Commons Attribution International License (CC BY).

http://creativecommons.org/licenses/by/4.0/

c) (i) Open Access

\section{Abstract}

Let $(M, F)$ be a compact Finsler manifold of hyperbolic type, and $\tilde{M}_{F}$ be its universal Finslerian covering. In this paper we show that the growth function of the volume of geodesic balls of $\tilde{M}_{F}$ is of purely exponential type.

\section{Keywords}

Finsler Manifold, Gromov Hyperbolic Manifold, Critical Exponent, Volume Entropy

\section{Introduction and Main Results}

A Finsler manifold $(M, F)$ is called of hyperbolic type, if there exists on the manifold $M$ a Riemannian metric $g_{0}$ of strictly negative curvature such that $F$ and $g_{0}$ are uniformly equivalent (cf. Definition 2.3).

We say that a function $f: \mathbb{R}_{+} \rightarrow \mathbb{R}_{+}$is of purely exponential type if there exist constants $a>1$ and $r_{0}>0$ such that

$$
\frac{1}{a} \leq \frac{f(r)}{\mathrm{e}^{h r}} \leq a \quad \forall r \geq r_{0},
$$

for some constant $h>0$. The real number $h$ is called the exponential factor of $f$. In 1969, Margulis (see [1]) proved, for suitable constant $h>0$ that

$$
a(p):=\lim _{r \rightarrow \infty} \frac{\operatorname{vol} S(p, r)}{\mathrm{e}^{h r}}
$$

exists at each point $p$ in manifolds of negative curvature and that the function $a$ is continuous. 
Clearly, this result implies purely exponential growth of volume of geodesic spheres. In 1979, Manning introduced a notion of volume entropy $h_{g}$ of a compact Riemannian manifold $(M, g)$ as follows (see [2]): if $\operatorname{vol}_{g}(p, r)$ denotes the volume of the geodesic ball $B_{g}(p, r)$ with centre $p$ and radius $r$ in the universal Riemannian covering $X$ of $(M, g)$,

$$
h_{g}:=\lim _{r \rightarrow \infty} \frac{\log \operatorname{vol} B_{g}(p, r)}{r},
$$

where the limit on the right hand side exists for all $p \in X$ and, in fact, is independent of $p$.

Manning showed that, in the case of non-positive curvature, $h_{g}$ coincides with the topological entropy.

In 1997, using the notions of Busemann density and Patterson Sullivan measure, G. Knieper proved the following result (see [3]):

If $\left(M, g_{0}\right)$ is a rank-1 compact Riemannian manifold of non-positive curvature and $X_{0}$ its universal Riemannian covering, there exist constants $a_{0} \geq 1$ and $r_{0} \geq 0$ such that

$$
\frac{1}{a_{0}} \leq \frac{\operatorname{vol} S_{g_{0}}(p, r)}{\mathrm{e}^{h_{g_{0}} r}} \leq a_{0} \quad \forall r \geq r_{0}
$$

Let $(M, g)$ be a compact Riemannian manifold of hyperbolic type without conjugate points, and $X$ be its universal Riemannian covering. In 2005, we show that the growth function of the volume of geodesic spheres of $X$ is of purely exponential type with the volume entropy $h_{g}$ as exponential factor (see [4]).

The main result of this paper is the following:

Theorem 1.1. Let $(M, F)$ be a compact Finsler manifold of hyperbolic type and $\tilde{M}_{F}$ be its universal Finslerian covering (cf. Definition 2.3). Let $h(F)$ be the volume entropy of $F$ (cf. Definition 2.1). Then, the growth function of the geodesic balls of $\tilde{M}_{F}$ is of purely exponential type with $h(F)$ as exponential factor.

Theorem 1.1 implies the following Corollary:

Corollary 1.2. Let $(M, F)$ be a compact Finsler manifold of hyperbolic type and $\tilde{M}_{F}$ be its universal Finslerian covering. Then, the critical exponent $\alpha^{F}$ (cf. Definition 4.2) of the group of the Deck transformations of $\tilde{M}_{F}$ is equal to the volume entropy $h(F)$ of $(M, F)$.

However, from Theorem 1.1, since all compact orientable surfaces of genus greater than one admits a metric $g_{0}$ of strictly negative curvature, we deduce the following properties:

Corollary 1.3. Let $M$ be a compact orientable surface of genus greater than one, $F$ a Finsler metric on $M$ and $\tilde{M}_{F}$ be its universal Finslerian covering. Then, the growth function of the geodesic balls of $\tilde{M}_{F}$ is of purely exponential type with $h(F)$ as exponential factor.

The paper is organized as follows: in Section 2, we recall some basic facts about the volume entropy of a compact Finsler manifold. Section 3 is devoted to the ideal boundary and the Gromov boundary of the universal Finslerian covering of a Finsler manifold of hyperbolic type. In Section 4, we introduce a notion of quasi-convex cocompact group and we provide the proof of the Theorem 1.1.

\section{The Volume Entropy of a Finsler Manifold of Hyperbolic Type}

In this section, we briefly recall some notions from Finsler geometry; see [5] or [6] and the references therein for more details. Let $M$ be a manifold and denote by $\pi: T M \rightarrow M$ the natural projection of $T M$ into $M$. A Finsler structure of $M$ is a function

$$
F: T M \rightarrow[0 ;+\infty)
$$

with the following properties:

1) $F$ is $C^{\infty}$ on the slit tangent bundle $T M \backslash\{0\}$;

2) $F(x, \lambda y)=\lambda F(x, y)$ for all $\lambda>0$;

3) The $n \times n$ Hessian matrix

$$
\left(g_{i j}\right):=\left(\left[\frac{1}{2} F^{2}\right]_{y^{i} y^{j}}\right)
$$

is positive definite at every point of $T M \backslash\{0\}$. 
Note that any Riemannian manifold $(M, g)$ is a Finsler manifold with $F=\|\|_{g}$. Let $\sigma:[a, b] \rightarrow M$ be a piecewise $C^{\infty}$ curve with velocity $\frac{\mathrm{d} \sigma}{\mathrm{d} t}=\frac{\mathrm{d} \sigma^{i}}{\mathrm{~d} t} \frac{\partial}{\partial x^{i}} \in T_{\sigma(t) M}$. Its length $l_{F}(\sigma)=\int_{a}^{b} F\left(\sigma, \frac{\mathrm{d} \sigma}{\mathrm{d} t}\right) \mathrm{d} t$. For $p$ and $x \in M$, denote by $C^{\infty}(p, x)$ the collection of all piecewise $C^{\infty}$ curves $\sigma:[a, b] \rightarrow M$ with $\sigma(a)=p$ and $\sigma(b)=x$. Define the metric distance from $p$ to $x$ by

$$
d_{F}(p, x)=\inf _{\sigma \in C^{\infty}(p, x)} l_{F}(\gamma) .
$$

Note that if $F$ is typically positively homogeneous (of degree 1 ) the distance $d_{F}$ is non-symmetric.

We say that the Finsler structure $F$ is absolute homogeneous is

$$
F(x, \lambda y)=|\lambda| F(x, y) \quad \text { for all } \lambda \in \mathbb{R} .
$$

In this case, the distance $d_{F}$ is symmetric. Let denote by $B_{F}^{+}(p, r)=\left\{x \in M: d_{F}(p, x)<r\right\}$ and $B_{F}^{-}(p, r)=\left\{x \in M: d_{F}(x, p)<r\right\}$. Every Finsler manifold comes with a natural volume form, which is described as follows:

Fix an arbitrary Riemannian metric $g$ on $M$ and let $d v_{g}$ be its volume form. Denote by $B_{g}(x, 1)$ and $B_{F}^{+} F(x, 1)$ the units balls of radius 1 with respect to $g$ and $F$ respectively, and let $\operatorname{vol}_{g} B_{g}(x, 1)$ and $\operatorname{vol}_{g} B_{F}^{+}(x, 1)$ be their volume with respect to $g$.

The Finsler form is given by

$$
d F(x)=\frac{\operatorname{vol}_{g} B_{g}(x, 1)}{\operatorname{vol}_{g} B_{F}^{+}(x, 1)} d v_{g},
$$

which is independant of the choice of the Riemannian metric $g$.

Definition 2.1. Let $(M, F)$ be a compact Finsler manifold and $\tilde{M}_{F}$ its universal Finslerian covering. The volume entropy of $F$ is defined by:

$$
h(F)=\lim _{r \rightarrow \infty} \frac{\log \operatorname{vol}_{g} B_{F}^{+}(x, r)}{r} .
$$

Definition 2.2. Let $(M, F)$ be a Finsler manifold.

1) A piecewise $C^{\infty}$ curve $c:[a, b] \rightarrow M$ satisfying $F(\dot{c})=1$ is said to be minimal if $l_{F}(c)=d_{F}(c(a), c(b))$.

2) A curve $c:[0, \infty) \rightarrow M$ is called a forward ray if $\left.c\right|_{[a, b]}$ is minimal for all $[a, b] \subset[0, \infty)$.

3) A curve $c:(\infty, 0] \rightarrow M$ is called a backward ray if $\left.c\right|_{[a, b]}$ is minimal for all $[a, b] \subset(\infty, 0]$.

4) A curve $c: \mathbb{R} \rightarrow M$ is called a minimal geodesic if $\left.c\right|_{[a, b]}$ is minimal for all $[a, b] \subset \mathbb{R}$.

Definition 2.3. Let $(M, F)$ be a Finsler manifold $M$. We say that $F$ is uniformly equivalent to a Riemannian metric $g$, if there is a constant $c_{F}$ such that

$$
\frac{1}{c_{F}} \cdot F \leq\|\cdot\| \leq c_{F} \cdot F
$$

Let $p: \tilde{M} \rightarrow M$ be the universal covering of $M$. Using the map $p$, we pull the Finsler structure $F$ back to $\tilde{M}$. The resulting $\tilde{F}$ defines on $T \tilde{M} \backslash \mathbf{0}$ a Finsler structure. We denote by $\tilde{M}_{F}$ the Finsler manifold $(\tilde{M}, \tilde{F})$. $\tilde{M}_{F}$ is the universal Finslerian covering of the Finsler manifold $(M, F)$

Let $\Gamma \subset I s o\left(\tilde{M}_{F}\right)$ be the group of deck transformations. We say that $F$ is invariant under $\Gamma$ if

$$
F(d \tau(p v) v)=F(v) \forall v \in T \tilde{M}, \tau \in \Gamma .
$$

Remark 2.4. Note that if $M$ is compact manifold and $F$ is invariant under the deck transformation $\Gamma$ then $F$ and $g$ are uniform equivalence.

\section{Ideal and Gromov Boundaries of Finsler Manifolds of Hyperbolic Type}

The following theorem is fundamental for the study of the ideal boundary of Finsler manifolds of hyperbolic type. It was proved by Morse in dimension 2 and by Klingenberg in arbitrary dimensions. The fact that the Morse Lemma also holds in Finsler case was first observed by E. M. Zaustinsky (see [7]). Due to Klingenberg 
(see [8]), the Morse Lemma holds in any dimension.

Theorem 3.1. (Morse Lemma, cf. [9]) Let $(M, F)$ be a Finsler manifold of hyperbolic type and $g_{0}$ be a metric of strictly negative curvature on $M$ such that $F$ and $g_{0}$ are uniformly equivalent and $\tilde{M}$ be the universal covering of $M$. Then there is a constant $r_{0}=r_{0}\left(F, g_{0}\right)>0$ with the following properties.

1) for any two points $x$ and $y \in \tilde{M}$, the $g_{0}$-geodesic-segment $\gamma:\left[0, d_{g_{0}}(x, y)\right] \rightarrow \tilde{M}$ from $x$ to $y$ and any F-minimal segment $c:\left[0, d_{F}(x, y)\right] \rightarrow \tilde{M}$ from $x$ to $y$ we have

$$
\max _{t \in\left[0, d_{F}(x, y)\right]} d_{g_{0}}\left(\gamma\left(\left[0, d_{g_{0}}(x, y)\right], c(t)\right)\right) \leq r_{0} .
$$

2) If $c:[0, \infty) \rightarrow \tilde{M}$ is a F-forward ray, then there exists a $g_{0}$-ray $\gamma:[0, \infty) \rightarrow \tilde{M}$ such that

$$
\sup _{t \in[0, \infty)} d_{g_{0}}(\gamma([0, \infty), c(t))) \leq r_{0} .
$$

These properties stay hold for F-backward rays and F-minimal geodesics.

Now let $(M, F)$ be a compact Finsler manifold of hyperbolic type and $\tilde{M}_{F}$ be its universal Finslerian covering. Let $g_{0}$ denote an associated metric of strictly negative curvature on $M$. Note that the universal Riemannian covering $\tilde{M}_{0}$ of $\left(M, g_{0}\right)$ is a Hadamard manifold and let denote by $\tilde{M}_{0}(\infty)$ its ideal boundary. Two $F$-forward rays $c$ and $c^{\prime}$ are said to be asymptotic if there exists a constant $D \geq 0$ such that $d_{H}\left(c\left(\mathbb{R}_{+}\right), c^{\prime}\left(\mathbb{R}_{+}\right)\right) \leq D$, where $d_{H}$ is the Hausdorff distance with respect to the distance $d_{F}$. This defines an equivalence relation on the set of $F$-forward rays of $\tilde{M}_{F}$. Let $\tilde{M}_{F}(\infty)$ be the coset of asymptotic $F$-forward rays $c$ of $\tilde{M}_{F}$. For each $F$-forward ray $c$ of $\tilde{M}_{F}$, it follows from Morse Lemma that there exists a $g_{0}$-geodesic ray $\gamma$ such that $d_{H}\left(c\left(\mathbb{R}_{+}\right), \gamma\left(\mathbb{R}_{+}\right)\right) \leq D$, where $D$ is the constant in Morse Lemma. Let $[c]$ be the equivalence class of a $F$-forward ray $c$ and let $[\gamma]$ the equivalence class of the $g_{0}$-geodesic $\gamma$. The map $f$ defined by

$$
\begin{aligned}
f: \tilde{M}_{F}(\infty) & \rightarrow \tilde{M}_{0}(\infty) \\
{[c] } & \mapsto[\gamma]
\end{aligned}
$$

is bijective. Then $f$ defines on $\tilde{M}_{F}(\infty)$ a natural topology with respect to which $\tilde{M}_{F}(\infty)$ and $\tilde{M}_{0}(\infty)$ are homeomorphic $\left(\tilde{M}_{F}(\infty) \simeq \tilde{M}_{0}(\infty)\right)$.

Let recall now some basic facts about Gromov hyperbolic spaces. Let $(X, d)$ be a metric space with a reference point $x_{0}$. The Gromov product of the points $x$ and $y$ of $X$ with respect to $x_{0}$ is the nonnegative real number $(x \cdot y)_{x_{0}}$ defined by:

$$
(x \cdot y)_{x_{0}}=\frac{1}{2}\left\{d\left(x, x_{0}\right)+d\left(y, x_{0}\right)-d(x, y)\right\} .
$$

Let $\delta \geq 0$. A metric space $(X, d)$ is said to be a $\delta$-hyperbolic space if

$$
(x \cdot y)_{x_{0}} \geq \min \left\{(x \cdot z)_{x_{0}} ;(y \cdot z)_{x_{0}}\right\}-\delta
$$

for all $x, y, z$ and every choice of reference point $x_{0}$. We call $X$ a Gromov hyperbolic space if it is a $\delta$-hyperbolic space for some $\delta \geq 0$. The usual hyperbolic space $\mathbb{H}^{n}$ is a $\delta$-hyperbolic space, where $\delta=\log 3$. More generally, every Hadamard manifold with sectional curvature $\leq-k^{2}$ for some constant $k>0$ is a $\delta$-hyperbolic space, where $\delta=k^{-1} \log 3$ (see [10] or [11]).

Lemma 3.2. (see [11] or [12]) Let $(X, d)$ be a complete geodesic $\delta$-hyperbolic space, $x_{0}$ a reference point in $X, x$ and $y$ two points of $X$. Then

$$
d\left(x_{0}, \gamma_{x y}\right)-4 \delta \leq(x \cdot y)_{x_{0}} \leq d\left(x_{0}, \gamma_{x y}\right)
$$

for every geodesic segment $\gamma_{x y}$ joining $x$ and $y$.

Definition 3.3. A function $f: \mathbb{R} \rightarrow \mathbb{R}$ is called $k$-convex if for all $x, y \in \mathbb{R}$, and $t \in[0,1]$,

$$
f(t x+(1-t) y) \leq t f(x)+(1-t) f(y)+k .
$$

Proposition 3.4. (see [11] or [12]) Let $(X, d)$ be a $\delta$-hyperbolic geodesic space and $c_{1}, c_{2}: \mathbb{R} \rightarrow X$ two minimizing geodesics. The function 


$$
\begin{aligned}
f: \mathbb{R} & \rightarrow \mathbb{R} \\
t & \mapsto d\left(c_{1}(t), c_{2}(t)\right)
\end{aligned}
$$

is $4 \delta$-convex.

Definition 3.5. Let $\left(X_{1}, d_{1}\right)$ and $\left(X_{2}, d_{2}\right)$ be two metric spaces. A map $\Phi: X_{1} \rightarrow X_{2}$ is called a quasiisometric map, if there exist constants $A>1$ and $\alpha>0$ with:

$$
\frac{1}{A} d_{1}(x, y)-\alpha \leq d_{2}(\Phi(x), \Phi(y)) \leq A d_{1}(x, y)+\alpha \quad \forall x, y \in X_{1} .
$$

In a metric space $X$, a quasi-geodesic (resp. quasi-geodesic ray) is a quasi-isometric map $\Phi: \mathbb{R} \rightarrow X$ (resp. $\left.\Phi: \mathbb{R}^{+} \rightarrow X\right)$.

Lemma 3.6. (see [11]) Let $X_{1}$ be a metric space and $\left(X_{2}, d_{2}\right)$ be a geodesic Gromov hyperbolic space. If there exists a quasi-isometric map $\Phi: X_{1} \rightarrow X_{2}$, then $X_{1}$ is also a Gromov hyperbolic space.

Now let $X$ be a Gromov hyperbolic manifold, $x_{0}$ a reference point in $X$. We say that the sequence $\left(x_{i}\right)_{i \in N}$ of points in $X$ converges at infinity if

$$
\lim _{i, j \rightarrow \infty}\left(x_{i} \cdot x_{j}\right)_{x_{0}}=\infty
$$

If $x_{1}$ is another reference point in $X$,

$$
(x \cdot y)_{x_{0}}-d\left(x_{0}, x_{1}\right) \leq(x \cdot y)_{x_{1}} \leq(x \cdot y)_{x_{0}}+d\left(x_{0}, x_{1}\right) .
$$

Then the definition of the sequence that converges at infinity does not depend on the choice of the reference point. Let us recall the following equivalence relation $\mathcal{R}$ on the set of sequences of points in $X$ that converge at infinity:

$$
\left(x_{i}\right) \mathcal{R}\left(y_{j}\right) \Leftrightarrow \lim _{i, j \rightarrow \infty}\left(x_{i} \cdot y_{j}\right)_{x_{0}}=\infty
$$

The Gromov boundary $X^{G}(\infty)$ of $X$ is the coset of sequences that converge at infinity.

Let $X$ be a simply connected manifold which is a Gromov hyperbolic space. One defines on the set $X \cup X^{G}(\infty)$ a topology as follows (see [11] page 22):

1) if $x \in X$, a sequence $\left(x_{i}\right)_{i \in N}$ converges to $x$ with respect to the topology of $X$.

2) if $\left(x_{i}\right)_{i \in N}$ defines a point $\xi \in X^{G}(\infty),\left(x_{i}\right)_{i \in N}$ converges to $\xi$.

3) For $\eta \in X^{G}(\infty)$ and $k>0$, let

$$
V_{k}(\eta):=\left\{y \in X \cup X^{G}(\infty) /(y \cdot \eta)_{x_{0}}>k\right\},
$$

where

$$
(x \cdot y)_{x_{0}}=\inf \left\{\liminf _{i \rightarrow \infty}\left(x_{i} \cdot y_{i}\right)_{x_{0}} / x_{i} \rightarrow x, y_{i} \rightarrow y\right\}
$$

for $x$ and $y$ elements of $X \cup X^{G}(\infty)$.

The set of all $V_{k}(\eta)$ and the open metric balls of $X$ generate a topology on $X \cup X^{G}(\infty)$. With respect to this topology, $X$ is dense in $X \cup X^{G}(\infty)$ and $X \cup X^{G}(\infty)$ is compact.

Lemma 3.7. (see [13]) Let $X$ be a $\delta$-hyperbolic space. Then

1) Each geodesic $\gamma: \mathbb{R} \rightarrow X$ defines two distinct points at infinity $\gamma(+\infty)$ and $\gamma(-\infty)$.

2) For each $(\eta, x) \in X^{G}(\infty) \times X$, there exists a geodesic ray $\gamma$ such that $\gamma(0)=x$ and $\gamma(+\infty)=\eta$. For any other geodesic ray $\gamma^{\prime}$ with $\gamma^{\prime}(0)=x$ and $\gamma^{\prime}(+\infty)=\eta$ we have $d\left(\gamma^{\prime}(t), \gamma(t)\right) \leq 4 \delta$ for all $t \geq 0$.

Definition 3.8. Let $\xi \in X^{G}(\infty)$ and $c: \mathbb{R}_{+} \rightarrow X$ be a minimal geodesic ray satisfying $c(+\infty)=\xi$. The function

$$
b_{c}(x):=\lim _{t \rightarrow \infty}(d(x, c(t))-t)
$$

is well-defined on $X$ and is called the Busemann function for the geodesic c.

Lemma 3.9. (see [13]) Let $X$ be a $\delta$-hyperbolic space, $\xi \in X^{G}(\infty), x, y \in X$ and c a geodesic ray with $c(0)=x$ and $c(+\infty)=\xi$. Then there exists a neighbourhood $\mathcal{V}$ of $\xi$ in $X \cup X^{G}(\infty)$ such that 


$$
\left|b_{c}(y)-(d(z, y)-d(z, x))\right| \leq K \text { for all } z \in \mathcal{V} \cap X,
$$

where $b_{c}$ is the busemann function for the geodesic $c$ and $K$ is a constant depending only on $\delta$.

Lemma 3.10. (see [11]) Let $X_{1}$ be a metric space and $\left(X_{2}, d_{2}\right)$ be a geodesic Gromov hyperbolic space. If there exists a quasi-isometric map $\phi: X_{1} \rightarrow X_{2}$, then $X_{1}$ is also a Gromov hyperbolic space. Moreover, if the map

$$
x \mapsto d_{2}\left(x, \phi\left(X_{1}\right)\right)
$$

is bounded above, $X_{1}^{G}(\infty) \simeq X_{2}^{G}(\infty)$ i.e. $X_{1}^{G}(\infty)$ is homeomorphic to $X_{2}^{G}(\infty)$.

The following lemma give an homeomorphism between the ideal boundary and the Gromov hyperbolic boundary of Hadamard manifolds:

Lemma 3.11. (see [14]) Let $X_{0}$ be a Hadamard manifold with sectional curvature $K_{X_{0}} \leq-k_{0}^{2}<0$ for some constant $k_{0}>0$. There exists a natural homeomorphism

$$
\phi: X_{0} \cup X_{0}^{G}(\infty) \rightarrow X_{0} \cup X_{0}(\infty) .
$$

In particular, $X_{0}^{G}(\infty) \simeq X_{0}(\infty)$.

Using Morse Lemma, (see Lemma 3.11) and the properties of the ideal boundaries, we obtain the following lemma:

Lemma 3.12. Let $(M, F)$ be a compact Finsler manifold of hyperbolic type and $\tilde{M}_{F}$ be its universal Finslerian covering. Let $g_{0}$ be an associated metric of strictly negative curvature on $M$ and $\tilde{M}_{0}$ be the universal Riemannian covering of $\left(M, g_{0}\right)$. We have

$$
\tilde{M}_{F}(\infty) \simeq \tilde{M}_{0}(\infty) \simeq \tilde{M}_{0}^{G}(\infty) \simeq \tilde{M}_{F}^{G}(\infty) .
$$

Proof. Since $\tilde{M}_{0}$ is a Hadamard manifold $K_{\tilde{M}_{0}} \leq-k_{0}^{2}<0$ for some constant $k_{0}>0$, it is a Gromov hyperbolic manifold and $\tilde{M}_{0}(\infty) \simeq \tilde{M}_{0}^{G}(\infty)$ (see Lemma 3.11). On the other hand, the fact that $F$ is uniformly equivalent to a Riemannian metric $g_{0}$ implies that $\tilde{M}_{F}$ is also a Gromov hyperbolic space and $\tilde{M}_{0}^{G}(\infty) \simeq \tilde{M}_{F}^{G}(\infty)$ (see Lemma 3.10). Finally, using the construction of the ideal boundary of $\tilde{M}_{F}$, we have $\tilde{M}_{F}(\infty) \simeq \tilde{M}_{0}(\infty)$.

\section{The Growth Rate of the Volume of Balls in Finsler Manifolds of Hyperbolic Type}

Definition 4.1. Let $X$ be a Gromov hyperbolic manifold with reference point $x_{0}$ and $\Gamma$ be a discrete and infinite subgroup of the isometry group Iso $(X)$ of $X$. For a given point $x \in X$, the limit set $\Lambda(\Gamma, x)$ is the set of the accumulation points of the orbit $\Gamma x$ in $X^{G}(\infty)$.

Definition 4.2. Let $(X, d)$ be a metric space and $\Gamma$ be a discrete and infinite subgroup of the isometry group Iso $(X)$ of $X$. For $x_{0}, x \in X$ and $s \in \mathbb{R}$,

$$
P_{s}\left(x, x_{0}\right):=\sum_{\gamma \in \Gamma} \mathrm{e}^{-s d\left(x, \gamma x_{0}\right)}
$$

denotes the Poincaré series associated to $\Gamma$. The number

$$
\alpha:=\inf \left\{s \in \mathbb{R} ; P_{s}\left(x, x_{0}\right)<\infty\right\}
$$

is called the critical exponent of $\Gamma$ and is independent of $x$ and $x_{0}$. The subgroup $\Gamma$ is called of divergence type if the Poincaré series diverge for $s=\alpha$. The following lemma introduces a useful modification (due to Patterson) of the Poincaré series if $\Gamma$ is not of divergence type.

Lemma 4.3. (see [15]) Let $\Gamma$ be a discrete group with critical exponent $\alpha$. There exists a function $f: \mathbb{R}_{+} \rightarrow \mathbb{R}_{+}$which is continuous, nondecreasing and such that

$$
\text { for all } a>0, \quad \lim _{r \rightarrow+\infty} \frac{f(r+a)}{f(r)}=1
$$

and the modified series

$$
\tilde{P}_{s}\left(x, x_{0}\right):=\sum_{\gamma \in \Gamma} f\left(d\left(x, \gamma x_{0}\right)\right) \mathrm{e}^{-d\left(x, \gamma x_{0}\right)}
$$


converges for $s>\alpha$ and diverges for $s \leq \alpha$.

Let now $(M, F)$ be a compact Finsler manifold of hyperbolic type, and $\tilde{M}_{F}$ be its universal Finslerian covering. Let $g_{0}$ denote a metric of strictly negative curvature on $M$. The universal covering $\tilde{M}_{0}$ of $\left(M, g_{0}\right)$ is a Hadamard manifold satisfying $K_{\tilde{M}_{0}} \leq-k_{0}^{2}<0$ for some constant $k_{0}>0$.

Let $\Gamma$ be the group of deck transformations of $\tilde{M}$ and $\alpha^{g_{0}}$ be its critical exponent with respect to the metric $g_{0}$. It follows from Theorem 5.1 in [3] that:

$$
\alpha^{g_{0}}=h\left(g_{0}\right):=\lim _{r \rightarrow \infty} \frac{\log \operatorname{vol}_{g_{0}} B_{g_{0}}(x, r)}{r} .
$$

The fact that $M$ is compact implies the existence of a constant $\lambda \geq 1$ such that

$$
\lambda^{-1} d_{g_{0}}(x, y) \leq d_{F}(x, y) \leq \lambda d_{g_{0}}(x, y) \text { for all } x, y \in \tilde{M} .
$$

Then, the critical exponent $\alpha^{F}$ of $\Gamma$ with respect to the metric $d_{F}$ belongs to $\left[\lambda^{-1} h\left(g_{0}\right), \lambda h\left(g_{0}\right)\right] \subset \mathbb{R}_{+}^{*}$.

Lemma 4.4. Let $(M, F)$ be a compact Finsler manifold of hyperbolic type, $\tilde{M}_{F}$ be its universal Finslerian covering and $\Gamma$ be the group of deck transformations of $\tilde{M}$. Then

1) $\Lambda^{F}(\Gamma, x)=\overline{\Gamma x} \cap \tilde{M}_{F}^{G}(\infty)$.

2) $\gamma\left(\Lambda^{F}(\Gamma, x)\right)=\Lambda^{F}(\Gamma, x)$ for all $\gamma \in \Gamma$ and $x \in \tilde{M}$.

3) $\Lambda^{F}(\Gamma, x)$ is independent of $x$.

4) $\Lambda^{F}(\Gamma, x)=\tilde{M}_{F}^{G}(\infty)$.

Proof of Lemma 4.4.

1) Direct because $\Lambda^{F}(\Gamma, x)=\overline{\Gamma x} \backslash \Gamma x$ and $\Gamma x \subset \tilde{M}$.

2) Let $\xi \in \Lambda^{F}(\Gamma, x)$. There exists a sequence $\gamma_{n} \in \Gamma$ such that $\lim _{n \rightarrow \infty} \gamma_{n} x=\xi$. Then $\lim _{n \rightarrow \infty} \gamma \cdot \gamma_{n} x=\gamma \xi$.

3) For all $\xi \in \Lambda^{F}(\Gamma, x)$, by the definition there is a sequence $\left(\gamma_{n}\right)_{n}$ of points of $\Gamma$ such that $\lim _{n \rightarrow \infty} \gamma_{n} x=\xi$. Then

$$
\lim _{m, n \rightarrow \infty}\left(\gamma_{n} x \cdot \gamma_{m} x\right)_{x_{0}}=\lim _{m, n \rightarrow \infty}\left[d_{F}\left(\gamma_{n} x, x_{0}\right)+d_{F}\left(\gamma_{m} x, x_{0}\right)-d_{F}\left(\gamma_{n} x, \gamma_{m} x\right)\right]=+\infty .
$$

For all $y \in \tilde{M}$, we have:

$$
\begin{aligned}
2\left(\gamma_{n} x \cdot \gamma_{n} y\right)_{x_{0}} & =d_{F}\left(\gamma_{n} x, x_{0}\right)+d_{F}\left(\gamma_{n} y, x_{0}\right)-d_{F}\left(\gamma_{n} x, \gamma_{n} y\right) \\
& =d_{F}\left(\gamma_{n} x, x_{0}\right)+d_{F}\left(\gamma_{n} y, x_{0}\right)-d_{F}(x, y) \\
& \geq d_{F}\left(\gamma_{n} x, x_{0}\right)-d_{F}(x, y)
\end{aligned}
$$

Hence,

$$
\lim _{n \rightarrow \infty}\left(\gamma_{n} x \cdot \gamma_{n} y\right)_{x_{0}}=+\infty \text { and } \lim _{n \rightarrow \infty} \gamma_{n} y=+\xi
$$

then $\xi \in \Lambda^{F}(\Gamma, y)$.

4) Let $g_{0}$ denotes a metric of strictly negative curvature on $M$. The universal Riemannian covering $\tilde{M}_{0}$ of $\left(M, g_{0}\right)$ is a Hadamard manifold satisfying $K_{\tilde{M}_{0} \leq-k_{0}^{2}<0}$ for some constant $k_{0}>0$. Then $\Lambda^{g_{0}}(\Gamma, x)=\tilde{M}_{0}(\infty)$ (see [3]). Since $\Gamma$ is cocompact, the identity map $I: \tilde{M}_{0} \rightarrow \tilde{M}_{F}$ defines a homeomorphism $I^{*}: \tilde{M}_{0}^{G}(\infty) \rightarrow \tilde{M}_{F}^{G}(\infty)$ (see Lemma 3.12). Let $\xi \in \tilde{M}_{F}^{G}(\infty)$ and $\eta \in \tilde{M}_{0}^{G}(\infty)$ such that $\xi=I^{*}(\eta)$. The fact that $\tilde{M}_{0}^{G}(\infty)=\Lambda^{g_{0}}(\Lambda)$, there is a sequence $\left(\gamma_{n}\right)_{n}$ in $\Gamma$ and $y \in \tilde{M}_{F_{*}}$ such that the sequence $\left(\gamma_{n} y\right)_{n}$ converges to $\eta$ in $\tilde{M} \cup \tilde{M}_{0}^{G}(\infty)$. Then $I\left(\gamma_{n} y\right)_{n}=\left(\gamma_{n} y\right)_{n}$ concerges to $I^{*}(\eta)=\xi$ in $\tilde{M}_{F} \cup \tilde{M}_{F}^{G}(\infty)$.

Let now $(X, g)$ be a Gromov hyperbolic manifold, and $\Gamma$ be a non trivial subgroup of $\operatorname{Iso}(X)$ and the limit set $\Lambda^{g}(\Gamma, x)$ of the orbit $\Gamma x$ in $X^{G}(\infty)$.

The gromov hull $E\left(\Lambda^{g}(\Gamma, x)\right)$ of $\Lambda^{g}(\Gamma, x)$ is the subset of $X$ defined by the collection of the images of the geodesics $c: \mathbb{R} \rightarrow X$ satisfying $c(-\infty) \in \Lambda^{g}(\Gamma, x)$ and $c(+\infty) \in \Lambda^{g}(\Gamma, x)$.

Definition 4.5. A non trivial subgroup $\Gamma$ of the isometry group Iso $(X)$ is quasi-convex cocompact if $E\left(\Lambda^{g}(\Gamma, x)\right) \backslash \Gamma$ is compact.

The following lemma is due to Coornaert (see [13]). 
Lemma 4.6. Let $(X, g)$ be a Gromov hyperbolic manifold with reference point $x_{0}$, and $\Gamma$ be a quasiconvex cocompact subgroup of the isometry group Iso $(X)$ with critical exponent $\alpha^{g}$. Then, for all $x \in X$, there is a constant $C_{x} \geq 1$ such that:

$$
\frac{1}{C_{x}} \mathrm{e}^{\alpha^{g_{r}}} \leq n_{\Gamma x}(r) \leq C_{x} \mathrm{e}^{\alpha^{g_{r}}}
$$

for all $r \geq 0$, where

$$
n_{\Gamma x}(r):=\operatorname{card}\left\{\gamma x \in \Gamma x ; d\left(\gamma x, x_{0}\right) \leq r\right\} .
$$

Proof of Theorem 1.1. By Lemma 4.4, we have $\Lambda^{F}(\Gamma, x)=\tilde{M}_{F}^{G}(\infty)$. Then, the Gromov hull $E\left(\Lambda_{\tilde{M}}^{F}(\Gamma, x)\right)$ of $\Lambda^{F}(\Gamma, x)$ is equal to $\tilde{M}_{F}$. This implies that $\Gamma$ is a quasi-convex cocompact subgroup of $\operatorname{Iso}\left(\tilde{M}_{F}\right)$.

For an orbit $\Gamma x$ of $\Gamma$ in $\tilde{M}_{F}$ we consider the map $K_{r}$ defined by:

$$
\begin{aligned}
K_{r}: \mathbb{R}_{+} & \rightarrow \mathbb{R}_{+} \\
x & \mapsto \begin{cases}1 & \text { if } 0 \leq x \leq r \\
0 & \text { if } x>r\end{cases}
\end{aligned}
$$

Let $\mathcal{F}$ be a fundamental domain of $\Gamma$ in $\tilde{M}_{F}$. We have:

$$
\begin{aligned}
\operatorname{vol}_{F}\left(B_{F}^{+}\left(x_{0}, r\right)\right) & =\int_{\tilde{M}} K_{r}\left(d_{F}\left(x_{0}, x\right)\right) d \operatorname{vol}_{F}(x) \\
& =\sum_{\gamma \in \Gamma} \int_{\gamma \mathcal{F}} K_{r}\left(d_{F}\left(x_{0}, x\right)\right) d \operatorname{vol}_{F}(x) \\
& =\sum_{\gamma \in \Gamma} \int_{\mathcal{F}} K_{r}\left(d_{F}\left(x_{0}, \gamma x\right)\right) d \operatorname{vol}_{F}(x) \\
& =\int_{\mathcal{F}} \sum_{\gamma \in \Gamma} K_{r}\left(d_{F}\left(x_{0}, x\right)\right) d \operatorname{vol}_{F}(x) \\
& =\int_{\mathcal{F}} n_{\Gamma x}(r) d \operatorname{vol}_{F}(x) .
\end{aligned}
$$

Let now $x_{1}$ be a fixed point in $\mathcal{F}$ and put $D=\operatorname{diam} \mathcal{F}$. For all $\gamma \in \Gamma$, and $x \in \mathcal{F}$, we have:

$$
d_{F}\left(\gamma x, x_{0}\right) \leq r \Rightarrow d_{F}\left(\gamma x_{1}, x_{0}\right) \leq r+D
$$

and for $r \geq D$,

$$
d_{F}\left(\gamma x_{1}, x_{0}\right) \leq r-D \Rightarrow d_{F}\left(\gamma x, x_{0}\right) \leq r
$$

Then,

$$
n_{\Gamma x_{1}}(r-D) \leq n_{\Gamma x}(r) \leq n_{\Gamma x_{1}}(r+D) \text { for all } x \in \mathcal{F} \text { and } r \geq D .
$$

By Lemma 4.6, there is a constant $C_{x_{1}} \geq 1$ such that:

$$
\frac{1}{C_{x_{1}}} \mathrm{e}^{\alpha^{F}(r-D)} \leq n_{\Gamma x}(r) \leq C_{x_{1}} \mathrm{e}^{\alpha^{F}(r+D)}
$$

for all $r \geq D$ and $x \in \mathcal{F}$. Then, there exist constants $a_{1}>1$ and $r_{1}:=D$ such that:

$$
\frac{1}{a_{1}} \leq \frac{\operatorname{vol}_{F}\left(B_{F}^{+}\left(x_{0}, r\right)\right)}{\mathrm{e}^{\alpha^{F} r}} \leq a_{1} \text { for all } r \geq r_{1} .
$$

\section{References}

[1] Margulis, M.A. (1969) Applications of Ergotic Theory to the Investigation of Manifolds of Negative Curvature. Functional Analysis and Its Applications, 3, 335-336. http://dx.doi.org/10.1007/BF01076325

[2] Manning, A. (1979) Topological Entropy for Geodesic Flows. Annals of Mathematics, 110, 567-573. http://dx.doi.org/10.2307/1971239

[3] Knieper, G. (2002) Hyperbolic Dynamics and Riemannian Geometry. In: Hasselblatt, B. and Katok, A., Eds., Hand- 
book of Dynamical Systems 1A, Elsevier Science, Amsterdam, 453-545.

[4] Ezin, J.-P. and Ogouyandjou, C. (2005) The Growth Function of the Volume of Geodesic Balls in Riemannian Manifolds of Hyperbolic Type. IMHOTEP, 6, 9-17.

[5] Bao, D., Chern, S.-S. and Shen, Z. (2000) An Introduction to Riemann-Finsler Geometry. Graduate Texts in Mathematics 200. Communication in Contemporay Math, No. 4, Springer-Verlag, 511-533.

[6] Shen, Z. (1977) Volume Comparison and Its Applications in Riemann-Finsler Geometry. Advances in Mathematics, 128, 306-328. http://dx.doi.org/10.1006/aima.1997.1630

[7] Zaustinsky, E.M. (1962) Extremals on Compact E-Surfaces. Transactions of the American Mathematical Society, 102, 433-445.

[8] Klingenberg, W. (1971) Geodätischer Fluss auf Mannigfaltigkeiten vom hyperbolishen Typ. Inventiones Mathematicae, 14, 63-82. http://dx.doi.org/10.1007/BF01418743

[9] Schröder, J.P. (2014) Minimal Rays on Surfaces of Genus Greater than One. arXiv:1404.0573v1 [math.DS]

[10] Ancona, A. (1988) Théorie du potentiel sur les graphes et les varieties. In: Ancona, A., et al., Eds., Potential Theory, Surveys and Problems, Lecture Notes in Mathematics, No. 1344, Springer-Verlag, Berlin.

[11] Coornaert, M., Delzant, T. and Papadopoulos, A. (1990) Géométrie et théorie des groups. Lecture Notes in Mathematics. No. 1441, Springer-Verlag, Berlin.

[12] Coornert, M. and Papadoupoulos, A. (1993) Symbolic Dynamics and Hyperbolic Groups. Lecture Notes in Mathematics, No. 1539, Springer-Verlag, Berlin.

[13] Coornaert, M. (1993) Mesures de Patterson-Sullivan sur le bord d'un espace hyperbolique au sens de Gromov. Pacific Journal of Mathematics, 159, 241-270. http://dx.doi.org/10.2140/pjm.1993.159.241

[14] Cao, J. (2000) Cheeger Isoperimetric Constants of Gromov Hyperbolic Spaces with Quasi-Pole. Communications in Contemporary Mathematics, 2, 511-533. http://dx.doi.org/10.1142/S0219199700000232

[15] Patterson, S. (1976) The Limit Set of Fuchsian Group. Acta Mathematica, 163, 241-273. http://dx.doi.org/10.1007/BF02392046 
Scientific Research Publishing (SCIRP) is one of the largest Open Access journal publishers. It is currently publishing more than 200 open access, online, peer-reviewed journals covering a wide range of academic disciplines. SCIRP serves the worldwide academic communities and contributes to the progress and application of science with its publication.

Other selected journals from SCIRP are listed as below. Submit your manuscript to us via either submit@scirp.org or Online Submission Portal.
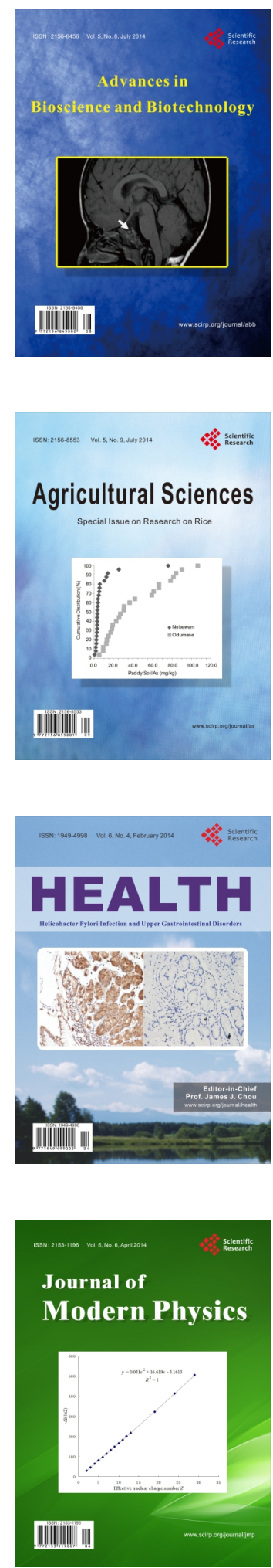
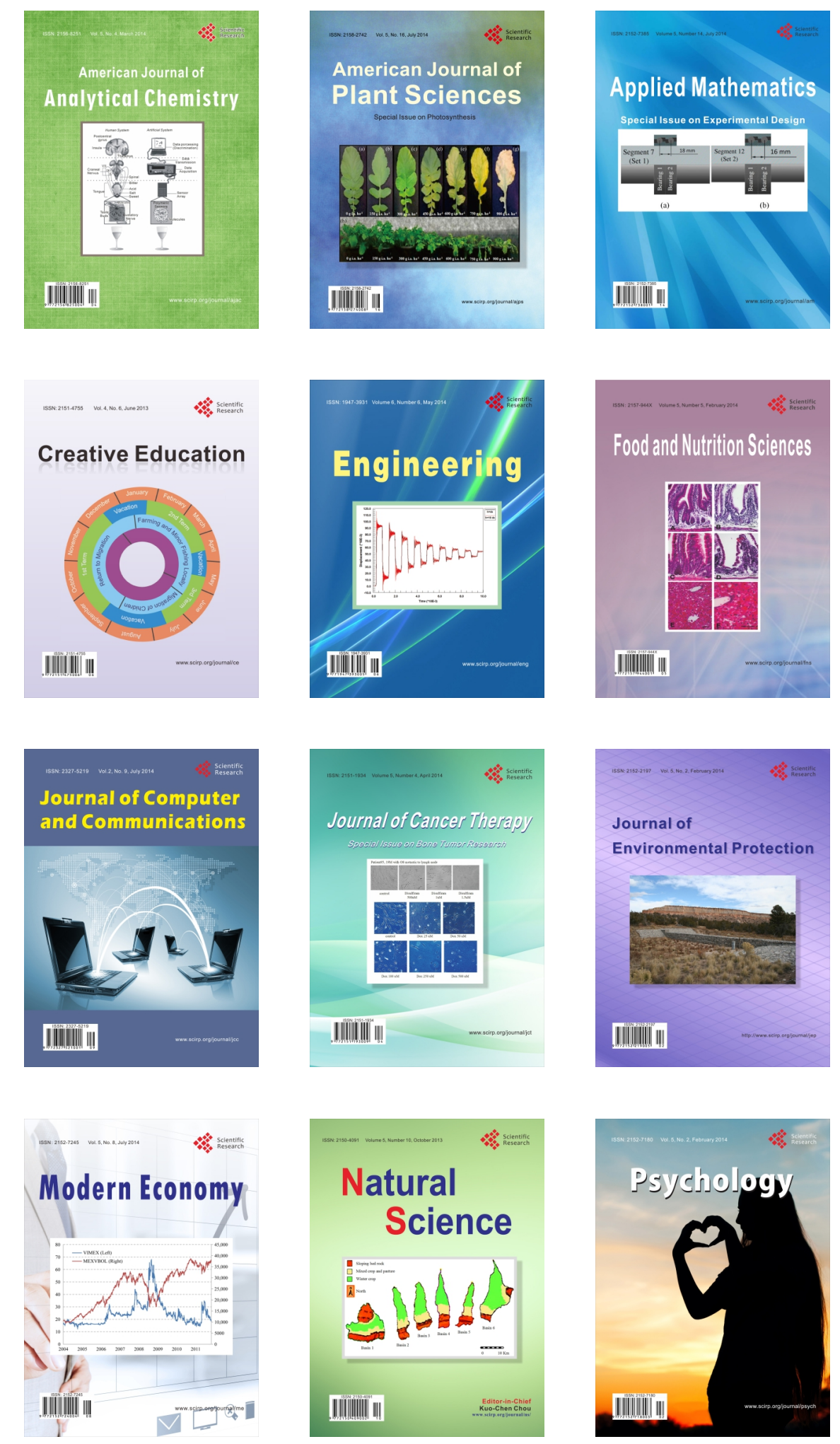